\title{
Genome-wide in vivo screen of circulating tumor cells identifies SLIT2 as a regulator of metastasis
}

Fan Xia ${ }^{1 \ddagger}$, Yuan Ma ${ }^{1 \ddagger}$, Kangfu Chen ${ }^{1}$, Bill Duong ${ }^{2}$, Sharif Ahmed ${ }^{1}$, Randy Atwal ${ }^{1}$, David Philpott $^{3}$, Troy Ketela ${ }^{4}$, Jennifer Pantea ${ }^{1}$, Sichun Lin ${ }^{1}$, Stephane Angers ${ }^{1,5 *}$,

Shana O. Kelley ${ }^{1,2,5,6 *}$

${ }^{1}$ Department of Pharmaceutical Sciences, University of Toronto, Toronto, Ontario, Canada. ${ }^{2}$ Department of Chemistry, University of Toronto, Toronto, Ontario, Canada. ${ }^{3}$ Department of Electrical and Computer Engineering, University of Toronto, Toronto, Ontario, Canada. ${ }^{4}$ Princess Margret Genomics Centre, University Health Network, Toronto, Ontario, Canada. ${ }^{5}$ Department of Biochemistry, University of Toronto, Toronto, Ontario, Canada. ${ }^{6}$ Institute for Biomaterials and Biomedical Engineering,

University of Toronto, Toronto, Ontario, Canada.

¥These authors contributed equally.

${ }^{\star}$ Corresponding authors. E-mail: shana.kelley@utoronto.ca, stephane.angers@utoronto.ca

\section{Abstract}

Circulating tumor cells (CTCs) break free from primary tumors and travel through the bloodstream and lymphatic system to seed metastatic tumors, which are the major cause of death from cancer. The identification of the major genetic factors that enhance production and persistence of CTCs in the bloodstream at a whole genome level would enable more comprehensive molecular mechanisms of metastasis to be elucidated and the identification of novel therapeutic targets, but this remains a challenging task due to the heterogeneity and extreme rarity of CTCs. Here, we describe the first in vivo genomewide CRISPR KO screen using CTCs directly isolated from a mouse xenograft. This screen elucidated SLIT2 - a gene encoding a secreted protein acting as a cellular migration cue - as the most significantly represented gene knockout in the CTC population. SLIT2 knockout cells are highly metastatic with hypermigratory and mesenchymal phenotype. Loss of SLIT2 is observed in human tumors, indicating its role as a negative modulator of tumor progression and metastasis. 


\section{Main}

Metastasis accounts for over $90 \%$ deaths from cancer, yet remains poorly understood and largely incurable ${ }^{1-3}$. Despite advances in cancer treatment that significantly reduce morbidity and mortality of various cancer types, metastasis still leads to poor prognosis of cancer patients and treating metastasis remains a tremendous challenge ${ }^{4,5} \mathrm{In}$ order to develop more effective therapies and improve patient outcomes, further progress elucidating the fundamental biology of metastasis is critical to reveal mechanisms at the molecular level and provide insights regarding new therapeutic targets. Comparing the genetic background between primary tumors and metastatic lesions using whole exome sequencing (WES) and whole genome analyses has facilitated the identification of genetic factors that drive tumor progression and dissemination ${ }^{6-8}$. However, deciphering drivers of metastasis solely based on the genetic information from solid tumors is limiting due to genetic divergence and tumor heterogeneity ${ }^{7-9}$.

As circulating tumor cells (CTCs) leave primary tumors and seed metastatic lesions, molecular characterization of CTCs is critical to facilitate comprehensive understanding of the metastatic processes. Progress in the area of CTC enrichment and single-cell sequencing technologies has enabled identification of CTC-specific mutations in cancer patients at the genomic level ${ }^{10-12}$. However, efficient capture of CTCs and their unbiased genomic amplification for WES are still challenging due to the rarity and fragility of CTCs $^{11-15}$. In addition, particular mutations observed in individual patient CTCs could be anecdotal, with low applicability and weak prognostic potential for large patient popualtions. Thus, new technologies and platforms are needed to comprehensively and systematically study the genetic factors underlying the CTC phenotypes that contribute to the metastatic process.

Here, we report a genome-wide CRISPR knockout (KO) screen designed to identify genes contributing to CTC dissemination in vivo. Xenografted tumors were seeded with pools of CRISPR-edited cells with each cell possessing loss-of-function of one gene in the human genome. Using a high-performance approach for CTC capture directly from 
blood coupled with next generation sequencing (NGS) of barcoded sgRNAs, gene knockouts that promoted CTC abundance were identified. This approach allowed the systematic identification of possible metastasis-promoting genetic factors with minimal bias. This strategy elucidated SLIT2 as a secreted factor that regulates epithelial-tomesenchymal transition (EMT) and drives the production of CTCs. Loss of SLIT2 increased CTC numbers and the extent of metastasis of prostate cancer in an animal model.

\section{Results}

\section{Genome-wide in vivo CRISPR-Cas9 knockout screen on CTCs}

Genome-wide CRISPR screens are powerful tools for the systematic and unbiased identification of genes involved in metastasis ${ }^{16-18}$. Combining a CRISPR-Cas9 genomewide editing system (TKOv3: Toronto KnockOut version 3 CRISPR library) ${ }^{19}$ and a highthroughput immunomagnetic microfluidic device for rare cell enrichment ${ }^{20,21}$, we systematically investigated genes promoting CTCs and tumor progression (Fig. 1a and Extended Data Fig. 1a). The in vivo genome-wide loss-of-function CRISPR-Cas9 screen was conducted using the prostate cancer cell line PC-3M as a model of tumor progression in immunodeficient mice. After subcutaneous injection of the cancer cells, tumor growth from the parental PC-3M cells or TKOv3-transduced PC-3M cells was monitored and compared for 3 weeks. No significant differences in the size of the primary tumors between these two groups were observed (Fig. 1b). At a predefined end point of 3 weeks, whole blood was collected from each animal to isolate CTCs for sgRNA enrichment analysis. Efficient isolation of CTCs from whole blood was accomplished by the immunomagnetic labeling of the epithelial marker EpCAM on the cell surface and magnetic deflection of CTCs away from blood cells using metglas tracks embedded in a microfluidic chip (Extended Data Fig. 1a, b) ${ }^{21}$. CTCs collected were lysed ${ }^{22}$ to obtain genomic DNA for the detection of sgRNA abundance using NGS analysis (Extended Data Fig. 1c). 
For sgRNA enrichment analysis, we first analyzed the TKOv3 library attrition across the initial CRISPR-edited PC-3M cells, the primary tumors, and the isolated CTCs. Compared to the sgRNA counts in the initial TKOv3-transduced PC-3M cell pool before injection, majority of the sgRNAs from the library had significantly reduced or no representation in the isolated CTC samples (Fig. 1c, d). Given that 30 million cells (each contained one sgRNA) were xenografted and a library size of 71,090 sgRNAs, an initial 400x representation of the TKOv3 library was obtained. With a median capture of 100 CTCs per animal, a drastic library attrition at the CTC level was expected. Notably, the reduction of the sgRNA representation was much less in the primary tumor samples since substantially more cells and input materials were available for NGS (Fig. 1c, d and Extended Data Fig. 2a). In addition, the overall distribution of sgRNAs in the CTC samples clustered tightly with each other and formed a clade distinct from the sgRNAs representing the CRISPR-edited PC-3M cells and primary tumor samples (Fig. 1e). When individual sgRNA enrichment was examined for the primary tumors, a clear trend emerged with the top-ranked genes representing well-established tumor suppressor genes (TSGs) such as TSC1 ${ }^{23,24}$. Frequent loss-of-function mutations of the top gene hits were also observed in patient tumor data collected from COSMIC with copy number variation analysis ${ }^{25,26}$ (Extended Data Fig. 3).

\section{Selection of top enriched sgRNAs in CTCs}

In order to analyze the CTC-enriched sgRNAs, we developed two selection criteria for robust candidate hit identification (Fig. $2 \mathrm{a}, 2 \mathrm{~b}, 2 \mathrm{c}$ ). In the first round of selection, we prioritized sgRNAs that were consistently enriched in 6 CTC samples isolated from individual mice. This resulted in the identification of $149 \mathrm{sgRNAs}$ that were enriched across all 6 CTC samples compared to their corresponding primary tumors (Fig. 2a). The second selection criterion takes into account the expected number of CTCs captured at the time of sample harvesting (median CTC counts 100). As a result, only the top 100 sgRNA counts from each of the 6 replicates were pooled together to generate a list of 162 unique sgRNAs (Fig. 2c). After filtering for sgRNAs that satisfied both selection criteria, the final CTC sgRNA enrichment list consisted of 38 unique sgRNAs and the genes targeted by these sgRNAs were selected for downstream analysis (Fig. 2b, d). The 
biological pathways represented by these 38 genes were found to be highly related to metastatic processes such as the regulation of apoptosis and cell proliferation (Fig. 2e).

\section{Functional validation of top hits with sub-library screen}

To functionally validate the top hits from the primary CTC CRISPR KO screen, a customized sub-pool CRISPR KO library consisting of 400 sgRNAs specifically targeting the 38 genes of interest (10 sgRNAs per gene) plus 20 non-targeted control sgRNAs was generated. CRISPR-edited PC-3M cells using the sub-pool library were xenografted using an analogous protocol to the original screen (Extended Data Fig. 1d). PC-3M cells were also engineered before the sub-pool screen for cell surface expression of GPI-anchored Myc-tag to allow Myc-based immunomagnetic capture of CTCs (Extended Data Fig. 1b). As observed previously, compared to the other sample groups (plasmid pool, infected cells before transplant, primary tumors), the number of unique sgRNAs with significant representation in the total sgRNA reads was dramatically reduced for the CTCs (Fig. 3a and Extended Data Fig. 2b). Notably, reads for the sgRNAs targeting SLIT2 accounted for $10 \%$ of the total sgRNA counts in the CTC samples, with 4 out of the 10 sgRNAs targeting SLIT2 highly represented in the CTC population (Fig. 3b). Accordingly, SLIT2 $\mathrm{KO}$ was ranked to be the most significant loss-of-function mutation that appeared to increase the number of CTCs (Fig. 3c). SLIT2 was thus selected for downstream analysis.

\section{Loss of SLIT2 is associated with poor prognosis of prostate cancer patients and induced metastasis in vivo}

SLIT2 belongs to a family of secreted proteins with a known role in axon guidance through interaction with the Roundabout (ROBO) family of receptors ${ }^{27,28}$. Recently, SLIT2 was shown to be expressed at different levels in various cancer tissues and to exhibit contextdependent promotion or inhibition of cancer progression ${ }^{29-33}$. Studies of the role of SLIT2 in prostate cancer are limited with contradictory findings reported in the literature ${ }^{34,35}$. In the light of our CTC CRISPR KO screen conducted with the prostate cancer cell line PC$3 \mathrm{M}$, we hypothesized that SLIT2 negatively regulates CTC production and thereby prevents metastatsis. 
Based on patient data collected from The Cancer Genome Atlas (TCGA), SLIT2 expression level is significantly reduced in tumor tissues compared to normal prostate tissues (Fig. 3d). Low SLIT2 expression is also associated with poor prognosis of prostate cancer patients (Fig. 3e). In addition, reduced expression of SLIT2 in tumor tissues compared to the corresponding normal tissues is observed in 26 out of the 31 human cancers recorded in TCGA (Extended Data Fig. 4), indicating the generality of the negative correlation between SLIT2 expression and development of tumors.

To investigate the role of SLIT2 in a prostate cancer model, SLIT2 KO PC-3M cells were generated using CRISPR editing to probe the effects of losing this gene in vivo (Extended Data Fig. 5a). Equal numbers of non-targeted control (NTC) or the SLIT2 KO PC-3M cells $\left(1 \times 10^{6}\right)$ were orthotopically injected into the prostate of immunodeficient mice. At the end point of tumor growth, the number of CTCs present in the whole blood of the mice injected with SLIT2 KO cells was increased up to fivefold compared to the group injected with the NTC cells (Fig. 4a and Extended Data Fig. 5d, e). The xenograft generated from SLIT2 KO cells also exhibited enhanced proliferation of cancer cells, resulting in significantly increased tumor size compared to the control group (Extended Data Fig. 5b, c). In order to assess if the enhanced CTC production in SLIT2 KO model was solely because of elevated tumor size, SLIT2 KO xenografts with comparable tumor sizes to the control were generated with a decreased number of SLIT2 KO cells at injection (Fig. 4b). In this case, SLIT2 KO again exhibited a significantly increased number of CTCs in whole blood compared to the control group (Fig. 4b), indicating enhanced intravasation and dissemination of cells lacking SLIT2. In addition, severe infiltration of neoplastic cells was observed inside the lymph nodes of the SLIT2 KO models, with cases of subcapsular and intravascular invasion found in multiple H\&E-stained histology sections (Fig. 4c, d). No invasion or peripheral infiltration of neoplastic cells was observed for the prostate cancer model generated from the control cells. We concluded that knockout of SLIT2 promotes CTC numbers and thereby increases metastatic burden in a prostate cancer model. 


\section{Loss of SLIT2 results in enhanced migratory and invasive behavior of prostate cancer cells}

To understand mechanisms by which SLIT2 modulates the metastatic process, we studied SLIT2 KO and NTC cells using a variety of phenotypic assays to assess cell proliferation, deformability, and invasion. 3D spheroids cultured from the SLIT2 KO and the NTC cells were comparable in size and composed of similar number of cells, but the SLIT2 KO spheroids appeared to be poorly organized with cells on the outer surface of the spheroids being loosely attached (Fig. 5a and Extended Data Fig. 6). To evaluate the impact of SLIT2 loss-of-function on the migratory potential of PC-3M cells, a specialized microfluidic device with migration channels connecting the cell loading and the nutrient holding pools was designed (Extended Data Fig. 7). The channels had a constant height of $5 \mu \mathrm{m}$, but decreasing width thereby requiring the cells to undergo remodelling to squeeze through narrowing channels towards the nutrients (Extended Data Fig. 7). While a comparable number of SLIT2 KO and NTC cells traveled through the migration channels wider than $10 \mu \mathrm{m}$, significantly more SLIT2 KO cells were able to fit into the channels of width $10 \mu \mathrm{m}$ or less (Fig. 5b, Extended Data Fig. 7b). The invasive behaviour of SLIT2 KO and control cells was also tested using a transwell cell invasion assay. A larger number of SLIT2 KO cells migrated from the top to the bottom of the membrane compared to the control group, with or without the presence of the chemoattractant in the lower chamber (Fig. 5c). Importantly, the number of invading SLIT2 KO cells was similar to control cells when the media was supplemented with exogenous SLIT2 protein (Fig. $5 c)$.

\section{Loss of SLIT2 results in activation of epithelial-to-mesenchymal transition}

The interrogation of a panel of cancer-related proteins using an oncology antibody array revealed several strong hits that connected the migratory and invasive phenotypes observed in SLIT2 KO cells to the epithelial-to-mesenchymal transition (EMT) (Fig. 5d and Extended Data Fig. 8). Protein levels of both Vimentin and Snail were enhanced in SLIT2 KO cells, which prompted us to hypothesize that the knockout produced a more 
mesenchymal phenotype. Reduced expression of the epithelial marker (E-cadherin) and increased expression of mesenchymal markers (Vimentin, N-cadherin, Snail) were observed for SLIT2 KO cells compared to NTC cells, indicating that EMT may be linked to the metastatic phenotype of cells lacking SLIT2 (Fig. 5e). Reversal of the mesenchymal phenotype was explored in a rescue experiment where exogenous SLIT2 was added to culture media and the level of Vimentin was analyzed. Addition of SLIT2 did not have a significant effect on Vimentin expression in control PC-3M cells (Fig. 5f). However, the level of Vimentin in SLIT2 KO PC-3M cells, which was originally doubled compared to the control cells, was significantly reduced with the addition of exogenous SLIT2 (Fig. 5f). In addition, we also tested Vimentin levels for a metastatic breast cancer cell line (MDA-MB231), and significant decrease of Vimentin expression was observed with the presence of SLIT2 (Fig. 5f), extending the link between SLIT2 and EMT in a different context.

\section{Loss of SLIT2 resulted in elevated complex I expression with hypersensitivity to rotenone}

To elucidate additional drivers of the invasive phenotype of SLIT2 KO cells, gene expression profiling of NTC and SLIT2 KO cells was also performed (Fig. 6a, b). Strikingly, SLIT2 KO cells exhibited increased expression of one component of the ATP synthase (ATP6) and multiple subunits of the complex I (MT-ND1, MT-ND2, MT-ND5) belonging to the mitochondrial electron transport chain (ETC) (Fig. 6b and Extended Data Fig. 9). Elevated expression of ETC components suggested a possible dependency of SLIT2 KO cells on mitochondrial activity and oxidative metabolism. Confirming this prediction, SLIT2 KO cells were significantly more sensitive to treatment with the complex I inhibitor rotenone when compared to the control cells (Fig. 6c). To test if the elevated activity of complex I contributed to the increased migratory potential of the PC-3M cells lacking SLIT2, we tested the ability of rotenone to block migration of SLIT2 KO and control cells using the microfluidic device described above (Extended Data Fig. 7). Upon treatment of rotenone, significant reduction of the number of deformed cells was observed in both groups at wider channel width (Fig. 6d). However, for the narrower channels, rotenone 
was more effective in inhibiting the migration of the SLIT2 KO cells than on the NTC cells (Fig. 6d).

\section{Discussion}

Previous studies of the role of SLIT2 in metastasis has produced contradictory observations in different experimental systems ${ }^{29,30,32-35}$. As a top hit in our genome-wide CTC CRISPR KO screen as well as in a sub-library screen, SLIT2 was identified as a strong modulator of invasiveness and metastasis. The metastasis-promoting effect of SLIT2 KO was also confirmed in a prostate cancer mouse model. No pathways have previously been connected with the invasive behaviour of prostate tumor cells carrying a loss-of-function mutation in SLIT2, except that the expression of SLIT2 is regulated by the transcriptional repressor EZH2 ${ }^{35}$. Our EMT and transcriptome studies revealed aberrant expression of multiple EMT markers and ETC components in SLIT2 KO cells that potentially contribute to metastasis. Mitochondrial activity has been linked to cancer progression in various studies ${ }^{36-40}$, with inhibitors of complex I reported to be promising anti-neoplastic therapies ${ }^{41,42}$. Our studies suggest that cancer cells lacking SLIT2 expression exhibit increased sensitivity towards complex I inhibition, revealing a potential novel therapeutic strategy for cancer patients with SLIT2 loss-of-function mutations.

Bulk sequencing of tumors has uncovered a number of cancer-causing mutations, some of which were harnessed for the development of new targeted therapeutic agents. The identification of gene mutations that are functionally implicated in tumor progression is paramount to nominate new targets for cancer. One important limitation is assessing the function of genes in rare cell populations, such as in CTCs or subsets of immune cells present in the tumor microenvironment. In this study, we performed for the first time an in vivo genome-wide CRISPR-Cas9 $\mathrm{KO}$ screen targeting rare CTC cells with prostate cancer origin. Development of this experimental pipeline is transferrable and can be easily applied to search for the unique CTC-promoting factors in other cancer types. This platform can also be further applied to explore genetic factors affecting other rare cells such as immune cells or other cell types composing the tumor microenvironment. Building on this study, we anticipate that combining efficient capture of rare cells with gene editing 
methodologies will provide new insights into the progression of various disease, including cancer, and will lead to the imagination of new therapies.

\section{Methods}

\section{Cell Culture}

Prostate cancer cell line PC-3M was obtained from Dr. Alison Allan at London Health Sciecnes, London, ON, Canada, and was cultured in RPMI-1640 (Wisent) supplemented with $10 \%$ fetal bovine serum (Wisent) and $1 \%$ penicillin/streptomycin (Wisent), at $37^{\circ} \mathrm{C}, 5 \% \mathrm{CO}_{2}$. MDA-MB-231 (ATCC) and lenti-X 293T cells (Takara Bio) were grown in DMEM (Sigma) supplemented with 10\% fetal bovine serum (Wisent), 4 $\mathrm{mM}$ stable glutamine (Sigma), and 1\% penicillin/streptomycin (Wisent), at $37{ }^{\circ} \mathrm{C}, 5 \%$ $\mathrm{CO}_{2}$.

\section{Lentiviral production}

All lentivirus was produced using lenti-X 293T cells. For TKOv3 genome-wide library production, $8.0 \mu \mathrm{g}$ TKOv3 pooled plasmid (Addgene) was co-transfected into $80 \%$ confluent lenti-X 293T cells with $4.8 \mu \mathrm{g}$ psPAX2 (Addgene) and 3.2 $\mu \mathrm{g}$ pMD2.G (Addgene) per $15 \mathrm{~cm}$ plate (Sarstedt). The mixture of plasmids (16.0 $\mu \mathrm{g}$ in $50 \mu \mathrm{L}$ $\mathrm{ddH}_{2} \mathrm{O}$ ) was added to $48 \mu \mathrm{L}$ of X-tremegene 9 DNA transfection reagents (Roche) that pre-incubated for $5 \mathrm{~min}$ in $750 \mu \mathrm{L}$ OptiMEM (Gibco). The mixture of plasmid-transfection reagent-OptiMEM was further incubated for $25 \mathrm{~min}$ before adding dropwise to lenti-X cells. $18 \mathrm{hr}$ later, media was changed to fresh DMEM. Viral particles were harvested 48 $\mathrm{hr}$ after the media exchange, snap freezed in liquid nitrogen, and stocked at $-80{ }^{\circ} \mathrm{C}$. The customized sub-library (plasmid pool synthesized at GenScript) lentivirus was prepared using the same protocol as the TKOv3 library. Individual SLIT2 CRISPR KO lentivirus was generated following the polyethyenimine (PEI) transfection protocol (Addgene). sgRNAs targeting the SLIT2 gene were cloned into the lentiCRISPRv2 (Addgene) and the 20 bp sgRNA sequences used were CGGGTTGGTCTGTACACTCA for KO 1 and 
ACGGAAAGCTTTCCTTGGGA for KO 2. For the non-targeted control, the sgRNA sequence used was CCCGAATCTCTATCGTGCGG.

\section{Lentiviral transduction and $\mathrm{MOI}$ determination}

Lentivirus was titrated into $2 \times 10^{5} \mathrm{PC}-3 \mathrm{M}$ cells on 6-well plates with $2 \mathrm{~mL}$ complete RPMI-1640 supplemented with $8 \mu \mathrm{g} / \mathrm{mL}$ polybrene (Sigma). After overnight infection, media was exchanged for fresh RPMl supplemented with $2 \mu \mathrm{g} / \mathrm{mL}$ puromycin (Sigma). After 48 hours of antibiotic selection, multiplicity of infection (MOI) was determined by comparing the number of cells remained at specific viral load with or without puromycin selection using $0.1 \%$ crystal violet staining (Sigma) and absorbance reading at $590 \mathrm{~nm}$. A MOI of 0.3 was used for the transduction of CRISPR libraries into the PC-3M cells.

\section{Genome-wide CTC CRISPR KO screen}

$1.0 \times 10^{8} \mathrm{PC}-3 \mathrm{M}$ cells were transduced with TKOv3 library (71,090 sgRNAs) at a MOI of 0.3 using polybrene as transduction reagent as described in the previous section. After 2 rounds of puromycin selection, $3 \times 10^{7}$ transduced cells were subcutaneously injected into the flank of each of the 6 male athymic nude (nu/nu) mice of 6-8 weeks old ( $1.5 \mathrm{x}$ $10^{7}$ each side per mouse). Tumor volume was measured twice a week using a caliper and calculated using the formula length $\mathrm{x}$ width ${ }^{2} / 2$. Mice were euthanized 3 weeks after transplant to collect tumor and whole blood samples (cardiac puncture). All animal work was conducted following the protocol approved by University of Toronto Animal Care Committee under the guidelines of Canadian Council on Animal Care (CCAC).

\section{PRISM chip design and fabrication}

An initial iteration of the PRISM chip ${ }^{21}$ was modified to increase throughput to $3 \times 10^{7}$ cells/hr/chip and sort cells into zero/low-, medium- and high- expression outlets.

Magnetically labeled cells are deflected laterally based on their level of expression. Deflection is facilitated using guides made from highly magnetically permeable alloy set at an angle from the fluid flow ( $5^{\circ}$ for medium and $20^{\circ}$ for high). In the presence of a 
magnetic field from a permanent magnet, localized regions of high magnetic flux are found at guide edges causing the attraction of magnetic nanoparticles ${ }^{43}$. A balancing of the magnetic force and the Stokes drag force from fluid flow causes magnetically labeled cells to follow the deflection guides until the angle changes or the guides end at the lateral edges of the device. Angle changes of the guides causes an increase in the component of the drag force and medium-expression cells to detach. Chips were fabricated on a soda lime glass wafer (UniversityWafer). Guides were patterned using photolithography and wet etching and channels were created using SU-8 photo resist (Kayaku AM). A PDMS slab (Dow Sylgard) was bonded to encapsulate the device according to an existing protocol ${ }^{44}$. Multiple chips were setup in parallel to simultaneously isolate CTCs from multiple blood samples. To prepare the PRISM chips for CTC isolation, the chips were primed with sterile water containing $0.1 \%$ Pluronic F68 (Sigma) under constant hydraulic pressure for overnight before use. The non-ionic surfactant Pluronic F68 prevented nonspecific capture of cells in the microchannel and the constant hydraulic pressure removed small air bubbles. For immunomagnetic-based cell sorting, the Prism chip was first mounted on a permanent magnet (BY088-N52, K\&J Magnetics). Before sample loading, a buffer solution made of HBSS (GIBCO) supplemented with $2 \%$ BSA (Sigma) and 5 mM EDTA (BioShop) was introduced to the Prism chip to remove the pluronic water.

\section{CTC collection and cell lysis}

To isolate the CTCs, each blood sample was diluted in a 1 to 1 ratio with the HBSS buffer solution and incubated with 10\% EpCAM MicroBeads (Miltenyi Biotec) with shaking at room temperature for an hour. Afterwards, equal volumes of buffer solution and the blood samples containing the magnetically labeled CTCs were loaded onto the buffer inlet and sample inlet respectively before withdrawn from the chip at a flow rate of $2 \mathrm{~mL} / \mathrm{hr}$. After sample processing, $300 \mu \mathrm{L}$ of buffer solution was loaded to both the sample and buffer inlets and withdrawn at the same flow rate to wash out remaining samples in the microchannels. Solution collected from the high-expression outlet ( 500 $\mu \mathrm{L}$ ) of each chip was injected into a $1.5 \mathrm{~mL}$ centrifuge microtube (Axygen) and set on the DynaMag ${ }^{\mathrm{TM}}-2$ magnet (ThermoFisher) for an hour. After the cells settled down, 
supernatant was pipetted out slowly without touching the bottom or the wall of the tube, leaving minimal solution $(5-10 \mu \mathrm{L})$ containing CTCs. Alkaline lysis method adapted from a previous publication was used to lyse CTCs ${ }^{22}$. Briefly, $10 \mu \mathrm{L} 200 \mathrm{mM} \mathrm{KOH}$ with 50 $\mathrm{mM}$ dithiothreitol was added to CTC solution and heated at $65^{\circ} \mathrm{C}$ for $10 \mathrm{~min}$ in a thermocycler. Lysate was then neutralized by $10 \mu \mathrm{L}$ of $900 \mathrm{mM}$ Tris- $\mathrm{HCl} \mathrm{pH} 8.3$ with 300 $\mathrm{mM} \mathrm{KCl}$ and $200 \mathrm{mM} \mathrm{HCl}$.

\section{Genomic DNA extraction and PCR amplification of sgRNA}

To extract gDNA from the CRISPR library-transduced cell pool (3-5 x $10^{7}$ cells) and the tumor tissues (100-200 mg), QIAamp DNA Blood Maxi Kit (Qiagen) was used following the manufacturer's instructions. For gDNA extraction from tumor tissues, tumors were chopped into small pieces using a razor blade and incubated with $1.8 \mathrm{~mL}$ tissue lysis buffer ATL (Qiagen) and $0.2 \mathrm{~mL}$ Proteinase K (Qiagen) at $56^{\circ} \mathrm{C}$ overnight before processed using the QIAamp DNA Blood Maxi Kit. gDNA extracted from the initial cell pool and the primary tumor samples were checked for 230/280 and 260/280 ratios before sending to the Princess Margret Genomics Center (PMGC, Toronto, ON Canada) for PCR amplification of the sgRNA region and generation of the barcoded sgRNA library. For the CTC samples, gDNA extraction was not necessary and $10 \mu \mathrm{L}$ of the neutralized lysate from each CTC sample was directly set for PCR amplification of the sgRNA region. Each $100 \mu \mathrm{L}$ PCR reaction was set as following:

$10 \mu \mathrm{L} 10$ x buffer (supplied with Ex Taq)

$8 \mu \mathrm{L}$ dNTP (supplied with Ex Taq)

$5 \mu \mathrm{L}$ Amp1 primer mix $(10 \mu \mathrm{M})$

$10 \mu \mathrm{L}$ neutralized CTC lysate

$65.5 \mu \mathrm{L}$ water

$1.5 \mu \mathrm{L}$ Ex Taq DNA polymerase (Takara Bio)

The thermocycling parameters used were:

Step 1: $95^{\circ} \mathrm{C}$ for $1 \mathrm{~min}$

Step 2: $95^{\circ} \mathrm{C}$ for $30 \mathrm{sec}$

Step 3: $52^{\circ} \mathrm{C}$ for $30 \mathrm{sec}$ 
Step 4: $72^{\circ} \mathrm{C}$ for $20 \mathrm{sec}$

Repeat steps $2-4$ for 34 additional times

Step 5: $72^{\circ} \mathrm{C}$ for $2 \mathrm{~min}$

Step 6: $4^{\circ} \mathrm{C}$ hold

PCR products were run on a $1 \%$ agarose gel to confirm the size of the product (expected size: $235 \mathrm{bp}$ ) before sent to PMGC for barcoded sgRNA library generation. NGS was done using Illumina NovaSeq 6000 with 1 million reads per sample.

\section{CTC sub-pool CRISPR KO screen}

Sub-library (400 sgRNAs) was synthesized and cloned into the same vector as the TKOv3 genome-wide library (lentiCRISPRv2) by GenScript. Cloned sub-library was deep sequenced to verify sgRNA representation in the plasmid pool. Lentivirus production, $\mathrm{MOI}$ determination, and in vivo screen were conducted in the same manner as the TKOv3 library, except that GPI-anchored Myc tag was lentivirally introduced to the PC-3M cells for displaying Myc on the cell surface before introducing the sub-library to the cells. Thus, CTCs in blood samples were labeled with $1 \%$ biotinylated anti-Myc antibody (Abcam, ab197139) for an hour and 5\% anti-biotin microbeads (Miltenyi Biotech) for an hour before being isolated on the Prism chip. Lysis and sgRNA PCR amplification of CTCs in the sub-library screen used the same protocol as the genomewide screen.

\section{NGS data and sgRNA enrichment analyses}

The genome-wide screen data was mapped by PMGC, and the customized sub-library data was mapped following the MAGeCK pipeline ${ }^{45}$. Number of reads in each sample was normalized by converting raw sgRNA counts to reads per million (rpm). The rpm values were then subject to log2 transformation for downstream analyses. To generate correlation heatmaps, NMF R package was used ${ }^{46}$ and Pearson correlations between individual samples were calculated using log2 rpm counts. Empirical cumulative distribution plot was generated using the ecdfplot function in the latticeExtra $R$ package. Biological pathway enrichment analysis was performed using Metascape ${ }^{47}$. For sgRNA 
enrichment analysis, fold change was obtained using MAGeCK and two criteria were used to identify the top candidate genes from the genome-wide screen based on CTC sgRNAs: 1) if a CTC sgRNA was enriched compared to its counterpart in the corresponding primary tumor sample (fold change>=1.0) across all the samples $(n=6)$; 2) if a sgRNA was counted as the top 100 in the normalized mapping results of each CTC sample. For the sub-library data, sgRNA counts were normalized and analyzed for enrichment using DrugZ ${ }^{48}$.

\section{In vivo validation of screen top hit}

PC-3M NTC or SLIT2 KO cells $\left(1.0 \times 10^{6}\right.$ each) were orthotopically injected into the prostate via the right dorsolateral lobe of 6-8 week-old male athymic nude (nu/nu) mice. After three weeks, mice were euthanized to collect primary tumor (size comparison), blood (CTC counting), and lymph nodes (metastasis histology). Lymph nodes for histology were formalin-fixed, paraffin-embedded, sectioned, and stained with hematoxylin and eosin (prepared by Pathology Research Program Laboratory at University Healthy Network). Prepared tissue sections were imaged and examined by a pathologist at Charles River laboratory for the assessment and grading of the metastatic lesions.

\section{Fabrication and performance of the 8-zone CTC capture device}

8-zone profiling chips were fabricated using 3D-stereolithography for the 3D printed molds and standard soft lithographic techniques for subsequent steps as described previously ${ }^{49-51}$. Prior to usage, the microfluidic chips were treated with $0.1 \%$ Pluronic F68 overnight. Devices were assembled with 2 rectangular arrays of N52 neodymium magnets above and below before connected to a syringe pump (Chemyx) set to withdraw for the duration of the sample processing. Blood samples were prepared following the same protocol as for the PRISM chips before loaded onto the 8-zone device, and CTCs were captured and profiled for EpCAM expression. 500 $\mu \mathrm{L}$ of PBS was withdrawn through the chip to displace the Pluronic F-68 solution before sample loading. The samples were processed at a flow rate of $750 \mu \mathrm{L} / \mathrm{hr}$. The samples were 
then fixed with $150 \mu \mathrm{L}$ of $4 \%$ paraformaldehyde (Sigma) in PBS, permeabilized with $150 \mu \mathrm{L}$ of $0.2 \%$ Triton-X-100 (Sigma) in PBS. The chips were washed in between each step with $125 \mu \mathrm{L}$ of CliniMACS PBS-EDTA solution (Miltenyi). Captured cells were immunostained by $200 \mu \mathrm{L}$ of an antibody cocktail containing $30 \mu \mathrm{g} / \mathrm{mL}$ anti-pan cytokeratin AF488 (Abcam, ab277270), $6 \mu \mathrm{g} / \mathrm{mL}$ anti-cytokeratin 18 FITC (LifeSpan BioSciences, LS-C46335), $30 \mu \mathrm{g} / \mathrm{mL}$ anti-cytokeratin 19 AF488 (BioLegend, 628508), and $3 \mu \mathrm{g} / \mathrm{mL}$ anti-mouse CD45 APC (BD Bioscience, 559864) in PBS supplemented with $1 \%$ BSA and $0.1 \%$ Tween 20 (Sigma) at a flow rate of $200 \mu \mathrm{L} / \mathrm{h}$. The captured cells were then stained with DAPI (Thermo Fisher, R37606) before fluorescently imaged and scanned using Nikon Ti-E Eclipse microscope with Andor's Neo sCMOS camera. CTCs were identified as nucleus present and EpCAM+ ${ }^{+} K^{+} \mathrm{CD}^{-} 5^{-}$cells.

\section{Spheroid assay}

3D spheroids consisted of NTC or SLIT2 KO cells were grown following a protocol described previously ${ }^{52}$. Briefly, RPMI-1640 was supplemented with $0.75 \%$ methylcellulose (Sigma), and the spheroids were cultured in pre-treated ultra-low attachment plates (Corning). To determine the amount of cells consisting the spheroids in each well, CellTiter-Glo 3D Cell Viability Assay (Promega) was used following manufacturer's instructions. Briefly, assay reagent was mixed vigorously with cell culture medium at a 1 to 1 ratio after the plate was brought to room temperature. After 25 min of incubation at room temperature, luminescence was recorded for each well.

\section{Migration chip fabrication and cell migration/deformability assay}

The migration chip includes a sample loading channel in the middle and two stimuli loading channels on each side. There are numerous narrow migration channels (constant height at $5 \mu \mathrm{m}$ ) with varying widths ranging from $6 \mu \mathrm{m}$ to $20 \mu \mathrm{m}$ connecting the sample channel and stimulation channels for cell migration. The chip consists of a PDMS substrate with microchannels and a glass cover. The PDMS substrate was fabricated using soft lithography. Briefly, the liquid base and reagent of PDMS was fully mixed at a ratio of $10: 1$ and casted on a SU8 negative photoresist-patterned silicon 
mold, and then incubated at $70^{\circ} \mathrm{C}$ for 2 hours for polymerization. The solidified PDMS was peeled off the mold and bonded with the glass cover upon plasma treatment. For cell deformability assay, the migration chip was first filled with $70 \%$ ethanol and UVtreated. The degassed and sterilized chip was then washed with PBS followed by cell culture medium. $3 \times 10^{6}$ PC-3M NTC or SLIT2 KO cells in $500 \mu \mathrm{L}$ complete medium were loaded to the sample channel and incubated at $37^{\circ} \mathrm{C}$ for overnight to allow cell attachment. The next day, the sample channel was washed with PBS and loaded with serum-free medium. The stimuli channels were filled with fresh cell culture medium with $10 \%$ FBS to produce nutrient gradient along the narrow migration channels. After 6 hours of incubation, cells across the entire chip were fluorescently labeled by loading 5 $\mu$ M SYTO 24 (Invitrogen) to both the sample and stimulation channels and incubate for 15 min. Migration behaviour of the cells were inspected under a fluorescence microscope. Cells that entered the migration channels or reached the stimuli channels were considered as deformed and migrated cells. The number of migrated cells was counted and compared for the deformability and migration potential of the cells from different samples. For testing the effect of rotenone on deformability and migration of the cells, rotenone at $\mathrm{IC}_{20}$ was added to the medium loaded onto the chip.

\section{Transwell cell invasion assay}

To prepare the invasion assay chambers, $750 \mu \mathrm{L}$ of $10 \%$ FBS-supplemented RPMI1640 was added to each of the 24 wells of the Transwell plate (Corning), and $100 \mu \mathrm{L}$ of Matrigel (Corning) was added to the cell culture inserts $(8 \mu \mathrm{m})$. The plate was incubated in a humidified incubator at $37^{\circ} \mathrm{C}$ with $5 \% \mathrm{CO}_{2}$ for an hour. $500 \mu \mathrm{L}$ of PC-3M NTC or SLIT2 KO cells in serum-free RPMI-1640 were added to the Matrigel-coated inserts at $1 \times 10^{5}$ cells $/ \mathrm{mL}$. Based on the conditions tested, media in the wells was either with or without $10 \%$ FBS and $1 \mu \mathrm{g} / \mathrm{mL}$ secreted N-SLIT2 (R\&D Systems, 8616-SL-050). The cells were incubated in the Matrigel-coated inserts for overnight in the incubator. The next day, medium was removed from the inserts and wells. The non-migrated cells on the upper side of the membrane was removed using a cotton swab and washed away with PBS. Cells remained on the membrane was fixed by submerging the membranes in the wells filled with cold methanol for $20 \mathrm{~min}$ and air-dried for $30 \mathrm{~min}$. The membranes 
were then submerged in $750 \mu \mathrm{L} 0.1 \%$ crystal violet (Sigma) in wells for $30 \mathrm{~min}$ at room temperature to stain the cells before washed thoroughly with distilled water. After airdrying, membranes were submerged in $750 \mu \mathrm{L}$ of $10 \%$ acetic acid (VWR) with shaking until completely dissolving the stain. Optical density of the dissolved stain in each well was determined at $590 \mathrm{~nm}$ using a microplate reader.

\section{Oncology proteome antibody array}

Oncology antibody array (R\&D Systems) was performed following the manufacturer's instructions. Briefly, NTC or SLIT2 KO cell lysates were diluted and incubated overnight with the nitrocellulose membranes that had been pre-spotted in duplicates with antibodies targeting 84 oncology-related proteins. The membranes were washed before incubated with a cocktail of biotinylated detection antibodies, followed by addition of Streptavidin-HRP. The amount of protein bound at each capture spot was detected using chemiluminescent substrate and the signal was captured using a ChemiDoc imaging system (Bio-Rad). Intensity of the capture spots were quantified using ImageJ.

\section{Immunoblot analysis}

Whole cell extracts were prepared with RIPA buffer (ThermoFisher) supplemented with protease inhibitor cocktail (Sigma). Proteins were separated on 4-15\% precast protein gels (Bio-Rad) and transferred onto PVDF membranes. Primary antibodies used were anti-SLIT2 (Abcam, ab134166), anti-Vimentin (Abcam, ab92547), anti-N-Cadherin (BD Bioscience, 610920), anti-E-cadherin (R\&D Systems, AF648), and anti-Snail (NEB, 3879S). Secondary antibodies were purchased from CST. Blots were visualized with chemiluminescent substrate (Thermo Scientific) using a ChemiDoc imaging system. Intensity of the protein bands were quantified using ImageJ.

\section{RNA-sequencing and data analysis}

RNA was extracted using RNeasy Plus Kit (Qiagen) following manufacturer's instructions and submitted to PMGC for cDNA library preparation and RNA sequencing 
using Illumina NovaSeq 6000. RNA-sequencing data was processed for sgRNA representation using $A^{*} S T A R$ procedure. Figures were generated using the normalized read counts in R and RStudio ( $R$ project, Revolution Analytics), Metascape, and the Gene Set Enrichment Analysis tools (Broad Institute).

\section{Cell viability assay}

NTC or SLIT2 KO PC-3M cells were seeded on 96-well plates in triplicates at a density of $2 \times 10^{4}$ cells per well. The next day, rotenone was titrated into the 96 -well plate at twofold serial dilution with concentration ranging from 0-10 $\mu \mathrm{M}$. Viable cell population was determined $48 \mathrm{hr}$ later using Cell Counting Kit-8 (Dojindo) following the manufacturer's instructions.

\section{Clinical and patient data analysis}

RNAseq and clinical data was retrieved from portals of TCGA, the Human Protein Atlas, GEPIA2, and COSMIC. SLIT2 expression median of 2.45 FPKM was used as the threshold to define low- or high-expression of SLIT2 in prostate cancer patients (PRAD). SLIT2 expression levels in tumor and normal tissues were retrieved for 31 cancer types to assess fold change. Information on the loss-of-function mutations in the top screen hits occurred in cancer patients was retrieved from Cancer Mutation Census (CMC) at COSMIC to compared with the level of loss-of-function mutations in well-known TSGs and OGs ${ }^{26}$. 


\section{References}

1. Steeg, P. S. Tumor metastasis: Mechanistic insights and clinical challenges. Nat. Med. 12, 895-904 (2006).

2. Chaffer, C. L. \& Weinberg, R. A. A perspective on cancer cell metastasis. Science (80-. ). 331, 1559-1564 (2011).

3. Fares, J., Fares, M. Y., Khachfe, H. H., Salhab, H. A. \& Fares, Y. Molecular principles of metastasis: a hallmark of cancer revisited. Signal Transduct. Target. Ther. 5, (2020).

4. Siegel, R. L., Miller, K. D. \& Jemal, A. Cancer statistics, 2020. CA. Cancer J. Clin. 70, 7-30 (2020).

5. Ganesh, K. \& Massagué, J. Targeting metastatic cancer. Nat. Med. 27, 34-44 (2021).

6. Priestley, P. et al. Pan-cancer whole-genome analyses of metastatic solid tumours. Nature 575, 210-216 (2019).

7. Beltran, $\mathrm{H}$. et al. Whole-exome sequencing of metastatic cancer and biomarkers of treatment response. JAMA Oncol. 1, 466-474 (2015).

8. Vignot, S., Besse, B., André, F., Spano, J. P. \& Soria, J. C. Discrepancies between primary tumor and metastasis: A literature review on clinically established biomarkers. Crit. Rev. Oncol. Hematol. 84, 301-313 (2012).

9. Turajlic, S. \& Swanton, C. Metastasis as an evolutionary process. Science (80-. ). 352, 169-175 (2016).

10. Lohr, J. G. et al. Whole-exome sequencing of circulating tumor cells provides a window into metastatic prostate cancer. Nat. Biotechnol. 32, 479-484 (2014).

11. Rossi, E. \& Zamarchi, R. Single-cell analysis of circulating tumor cells: How far have we come in the-omics era? Front. Genet. 7, 1-12 (2019).

12. Alix-Panabières, C. \& Pantel, K. Challenges in circulating tumour cell research. Nat. Rev. Cancer 14, 623-631 (2014).

13. Ortiz, V. \& Yu, M. Analyzing Circulating Tumor Cells One at a Time. Trends Cell Biol. 28, 764-775 (2018).

14. Zhu, Z., Qiu, S., Shao, K. \& Hou, Y. Progress and challenges of sequencing and analyzing circulating tumor cells. Cell Biol. Toxicol. 34, 405-415 (2018).

15. Labib, M. \& Kelley, S. O. Circulating tumor cell profiling for precision oncology. Mol. Oncol. 1-25 (2021) doi:10.1002/1878-0261.12901.

16. Chow, R. D. \& Chen, S. Cancer CRISPR Screens In Vivo. Trends in Cancer 4, 349-358 (2018).

17. Chen, S. et al. Genome-wide CRISPR screen in a mouse model of tumor growth and metastasis. Cell (2015) doi:10.1016/j.cell.2015.02.038.

18. Ebright, R. Y. et al. Deregulation of ribosomal protein expression and translation promotes breast cancer metastasis. Science (80-. ). (2020) doi:10.1126/science.aay0939.

19. Hart, T. et al. Evaluation and design of genome-wide CRISPR/SpCas9 knockout screens. G3 Genes, Genomes, Genet. 7, 2719-2727 (2017).

20. Mair, B. et al. High-throughput genome-wide phenotypic screening via immunomagnetic cell sorting. Nat. Biomed. Eng. (2019) doi:10.1038/s41551-0190454-8.

21. Aldridge, P. M. et al. Prismatic Deflection of Live Tumor Cells and Cell Clusters. 
ACS Nano (2018) doi:10.1021/acsnano.8b07616.

22. Kim, S. A. et al. An efficient and reliable DNA extraction method for preimplantation genetic diagnosis: a comparison of allele drop out and amplification rates using different single cell lysis methods. Fertil. Steril. (2009) doi:10.1016/j.fertnstert.2008.06.033.

23. Brugarolas, J. Regulation of mTOR function in response to hypoxia by REDD1 and the TSC1/TSC2 tumor suppressor complex. Genes Dev. 18, 2893-2904 (2004).

24. Inoki, K., Li, Y., Zhu, T., Wu, J. \& Guan, K.-L. TSC2 is phosphorylated and inhibited by Akt and suppresses mTOR signalling. Nat. Cell Biol. 4, 648-657 (2002).

25. Sondka, Z. et al. The COSMIC Cancer Gene Census: describing genetic dysfunction across all human cancers. Nat. Rev. Cancer 18, 696-705 (2018).

26. Sanchez-Vega, F. et al. Oncogenic Signaling Pathways in The Cancer Genome Atlas. Cell 173, 321-337.e10 (2018).

27. Rothberg, J. M., Hartley, D. A., Walther, Z. \& Artavanis-Tsakonas, S. slit: An EGFhomologous locus of D. melanogaster involved in the development of the embryonic central nervous system. Cell 55, 1047-1059 (1988).

28. Manavalan, M. A., Jayasinghe, V. R., Grewal, R. \& Bhat, K. M. The glycosylation pathway is required for the secretion of Slit and for the maintenance of the Slit receptor Robo on axons. Sci. Signal. 10, 1-14 (2017).

29. Tong, M., Jun, T., Nie, Y., Hao, J. \& Fan, D. The role of the SLIT/Robo signaling pathway. J. Cancer 10, 2694-2705 (2019).

30. Jiang, Z. et al. Targeting the SLIT/ROBO pathway in tumor progression: molecular mechanisms and therapeutic perspectives. Ther. Adv. Med. Oncol. 11, 1-14 (2019).

31. Wang, L. J. et al. Targeting Slit-Roundabout signaling inhibits tumor angiogenesis in chemical-induced squamous cell carcinogenesis. Cancer Sci. 99, 510-517 (2008).

32. Jeon, M. J. et al. The role of Slit2 as a tumor suppressor in thyroid cancer. Mol. Cell. Endocrinol. 483, 87-96 (2019).

33. Tavora, B. et al. Tumoural activation of TLR3-SLIT2 axis in endothelium drives metastasis. Nature 586, 299-304 (2020).

34. Bartholow, T. L., Becich, M. J., Chandran, U. R. \& Parwani, A. V. Immunohistochemical staining of Slit2 in primary and metastatic prostatic adenocarcinoma. Transl. Oncol. 4, 314-320 (2011).

35. Yu, J. et al. The neuronal repellent SLIT2 is a target for repression by EZH2 in prostate cancer. Oncogene 29, 5370-5380 (2010).

36. Porporato, P. E. et al. A mitochondrial switch promotes tumor metastasis. Cell Rep. 8, 754-766 (2014).

37. Porporato, P. E., Filigheddu, N., Pedro, J. M. B. S., Kroemer, G. \& Galluzzi, L. Mitochondrial metabolism and cancer. Cell Res. 28, 265-280 (2018).

38. Scheid, A. D., Beadnell, T. C. \& Welch, D. R. Roles of mitochondria in the hallmarks of metastasis. Br. J. Cancer 124, 124-135 (2021).

39. Bhandary, B., Marahatta, A., Kim, H. R. \& Chae, H. J. Mitochondria in relation to cancer metastasis. J. Bioenerg. Biomembr. 44, 623-627 (2012). 
40. Lebleu, V. S. et al. PGC-1a mediates mitochondrial biogenesis and oxidative phosphorylation in cancer cells to promote metastasis. Nat. Cell Biol. 16, 9921003 (2014).

41. Urra, F. A., Muñoz, F., Lovy, A. \& Cárdenas, C. The mitochondrial Complex(I)ty of cancer. Front. Oncol. 7, 1-8 (2017).

42. Naguib, A. et al. Mitochondrial Complex I Inhibitors Expose a Vulnerability for Selective Killing of Pten-Null Cells. Cell Rep. 23, 58-67 (2018).

43. Shevkoplyas, S. S., Siegel, A. C., Westervelt, R. M., Prentiss, M. G. \& Whitesides, G. M. The force acting on a superparamagnetic bead due to an applied magnetic field. Lab Chip 7, 1294-1302 (2007).

44. Philpott, D. et al. High-throughput microfluidic cell sorting platform (MICS). 1-13 (2019) doi:10.21203/rs.2.10282/v1.

45. Wang, B. et al. MAGeCK Flute. 14, (2019).

46. Gaujoux, R. \& Seoighe, C. A flexible R package for nonnegative matrix factorization. BMC Bioinformatics 11, (2010).

47. Zhou, Y. et al. Metascape provides a biologist-oriented resource for the analysis of systems-level datasets. Nat. Commun. 10, (2019).

48. Colic, M. et al. Identifying chemogenetic interactions from CRISPR knockout screens with drugZ. Genome Med. 232736 (2019) doi:10.1101/232736.

49. Labib, M. et al. Tracking the expression of therapeutic protein targets in rare cells by antibody-mediated nanoparticle labelling and magnetic sorting. Nat. Biomed. Eng. 5, 41-52 (2021).

50. Duong, B. T. V. et al. A liquid biopsy for detecting circulating mesothelial precursor cells: A new biomarker for diagnosis and prognosis in mesothelioma. EBioMedicine 61, (2020).

51. Wang, Z. et al. Ultrasensitive and rapid quantification of rare tumorigenic stem cells in hPSC-derived cardiomyocyte populations. Sci. Adv. 6, 1-12 (2020).

52. Han, K. et al. CRISPR screens in cancer spheroids identify 3D growth-specific vulnerabilities. Nature (2020) doi:10.1038/s41586-020-2099-x. 

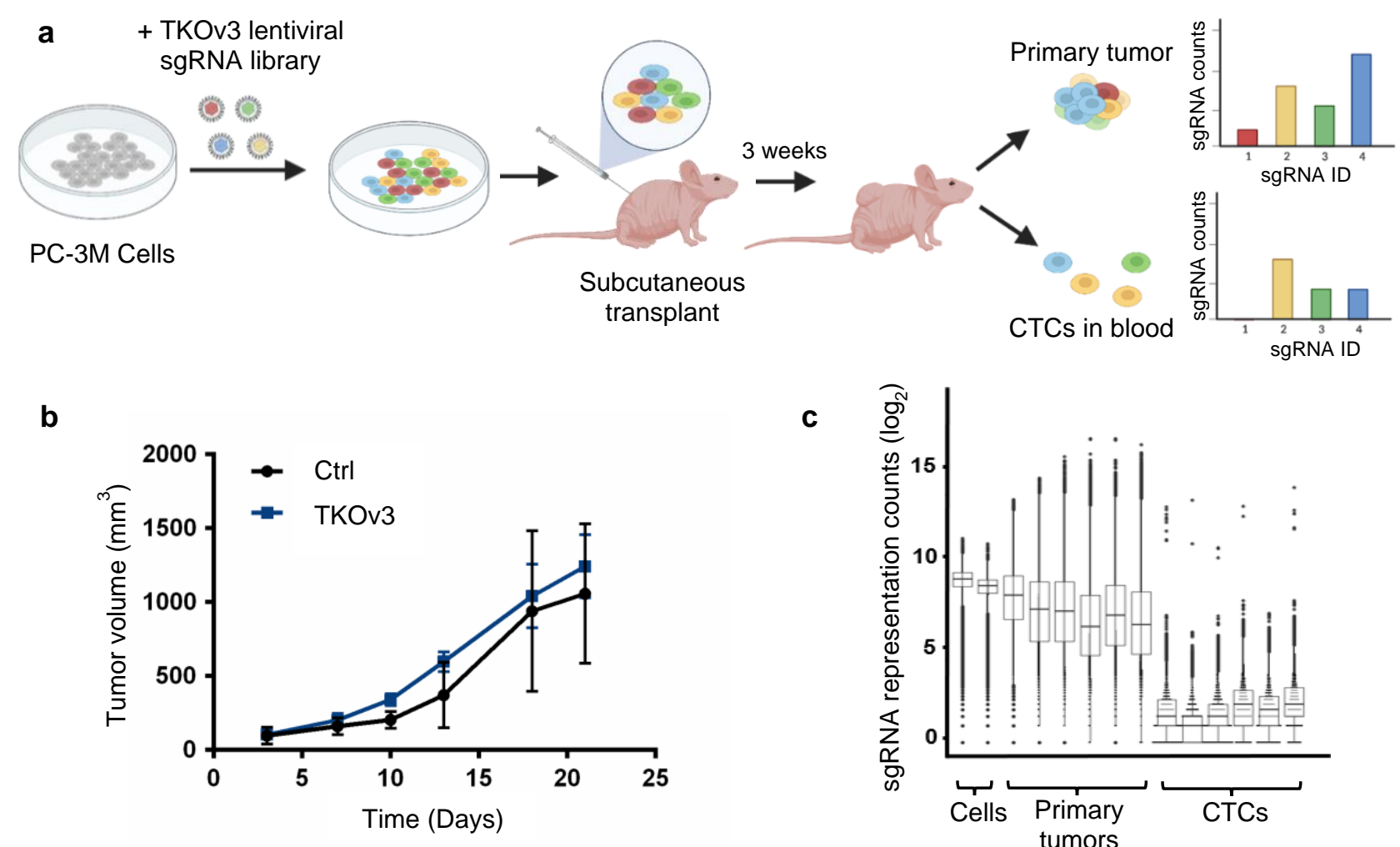

d
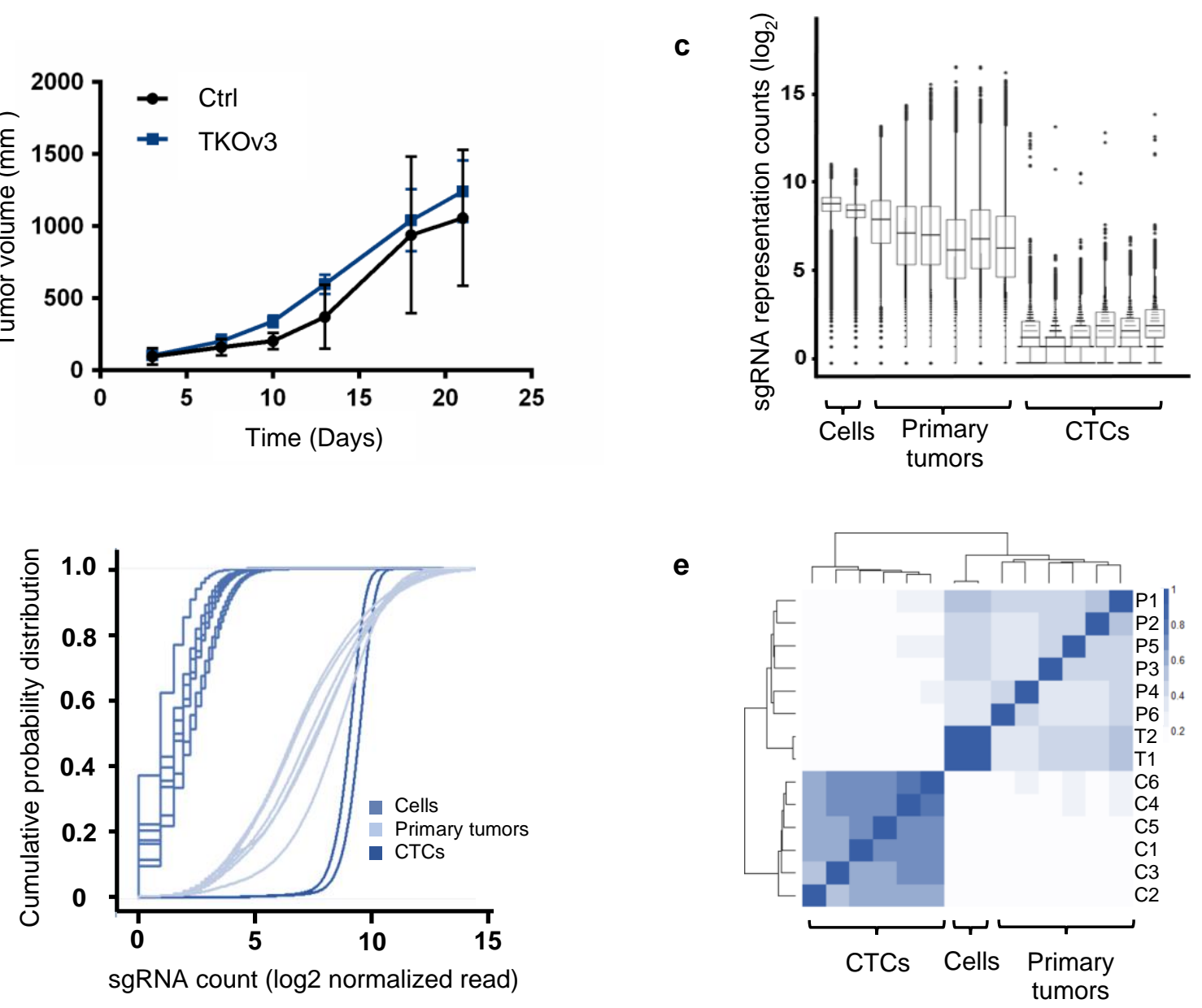

Figure 1: An in vivo genome-wide CRISPR-Cas9 knockout screen identifying CTCpromoting genetic factors. a. Experimental design of the genome-wide CRISPR-Cas9 KO screen focused on CTCs. b. Primary tumour growth curves of the immunocompromised mice subcutaneously transplanted with either the TKOv3-transduced PC-3M cells, or the control cells ( $n=3$ for each group). Error bars indicate SD. c. Box plot of the sgRNA counts from the TKOv3-transduced cell pool before transplantation (Cells), primary tumours, and CTCs. d. Cumulative probability distribution of sgRNA counts from the TKOv3-transduced cell pool before transplantation (Cells), primary tumours, and CTCs. e. Pearson correlation coefficient of the sgRNA counts from the TKOv3-transduced cell pool before transplantation $(\mathrm{Tn})$, primary tumours $(\mathrm{Pn})$, and CTCs $(\mathrm{Cn})$. 
a

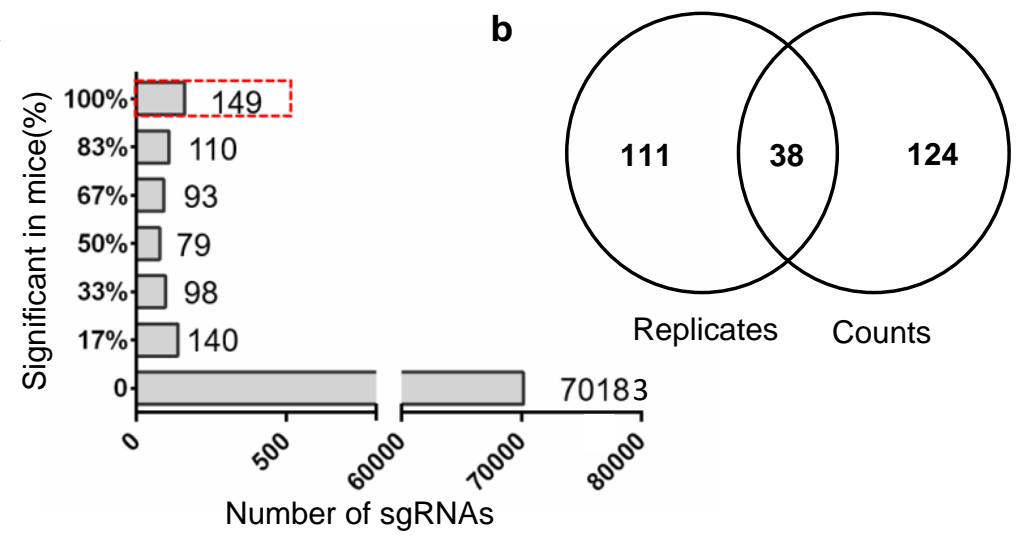

b

70183
C

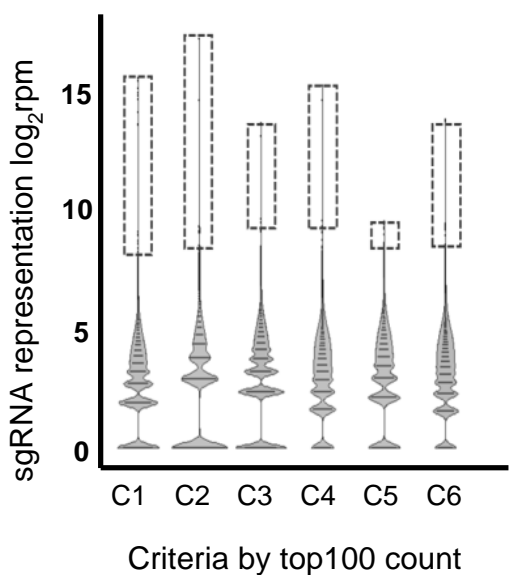

d

\begin{tabular}{|c|c|c|c|c|}
\hline ADRB2 & CLMP & GLB1L3 & PHACTR1 & SLIT2 \\
\hline AMBRA1 & COL18A1 & GRIA3 & PPAP2A & SUCLG2 \\
\hline ANKRD44 & CUL1 & IDI2 & RGS8 & TTC13 \\
\hline ANXA5 & CUL4B & IL12B & RORC & TTK \\
\hline ARRB2 & EHHADH & MAP3K12 & SCEL & ZCCHC16 \\
\hline ATAD2 & ENGASE & NDUFA1 & SERPINF1 & ZNF597 \\
\hline C9orf142 & ERC1 & NDUFA12 & SLC10A6 & \\
\hline CBS & FUT8 & OGDHL & SLC47A2 & \\
\hline
\end{tabular}

e

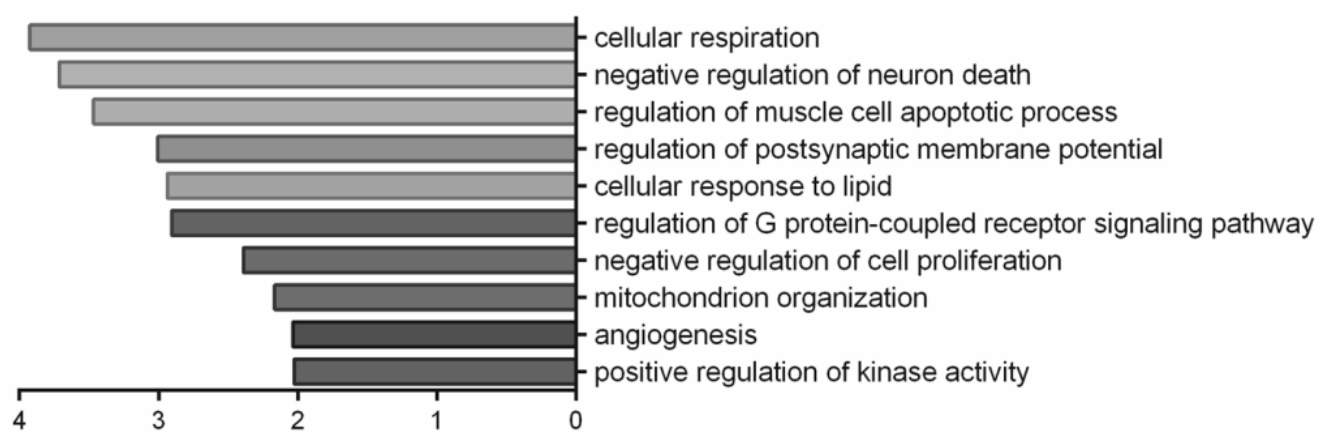

Figure 2: Identification of top hits from the genome-wide CRISPR-Cas9 KO screen. a. Frequency (percent of mice) of each sgRNA being enriched in CTCs compared to the corresponding primary tumours. The dashed box highlights the 149 sgRNAs enriched in CTCs across all 6 mice. b. Venn diagram showing selection of the top-enriched sgRNAs from the CTC samples. 38 sgRNAs satisfied both the "counts" criteria in (c) and the "replicates" criteria in (a), and their targeted genes were selected for downstream analysis. c. Violin plots of the sgRNA counts from the CTC samples. The dashed boxes highlight the top 100 sgRNA counts from each CTC sample. d. List of the 38 genes selected for the construction of the sub-library. e. Biological pathways being enriched from the 38 genes selected. 
a

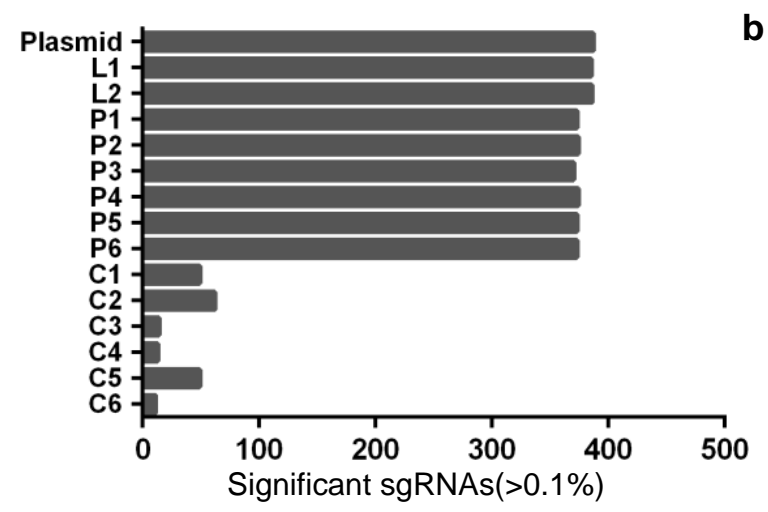

b

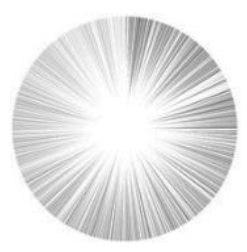

Cell pool

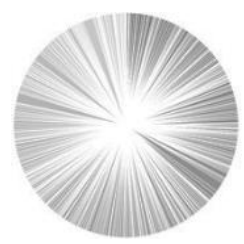

Primary tumor

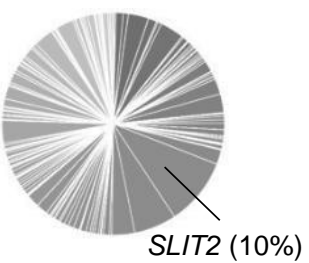

CTC

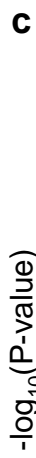

\section{d}

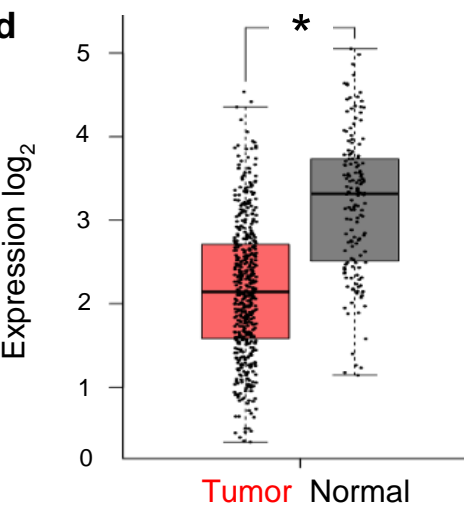

e

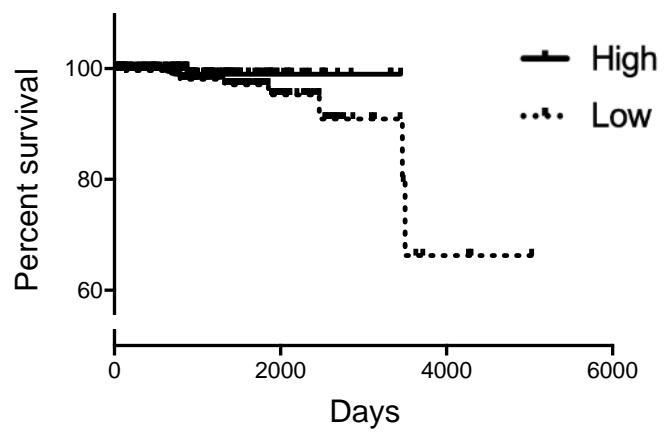

Figure 3: Loss of SLIT2 was a key factor of CTC production in the sub-pool screen and was associated with poor prognosis of prostate cancer patients. a. Bar graph of the number of significant sgRNAs (counts $>0.1 \%$ of total reads) from the plasmid library (Plasmid), the initial cell pool before transplant $(\mathrm{Ln})$, primary tumors $(\mathrm{Pn})$, and CTCs $(\mathrm{Cn})$ of the sub-library CRISPR KO screen. b. Pie chart showing gene enrichment (percentage of total reads) of the initial cell pool, primary tumor, and CTC samples from the sub-pool CRISPR KO screen. c. Gene ranking from the sub-pool CRISPR KO screen. SLIT2 KO was found to be the most abundant gene KO present in CTCs. d. SLIT2 expression levels in tumor and normal tissues of prostate adenocarcinoma (PRAD) patients were compared using RNAseq data collected from TCGA and prepared by GEPIA2. (Tumor: $n=492$; Normal: $n=152$ ) e. Survival plot of PRAD patients with low or high expression of SLIT2. Data was collected from TCGA, with an expression cut-off at 2.45 FPKM. (High expression: $\mathrm{n}=189$; Low expression: $\mathrm{n}=305$ ) 
a

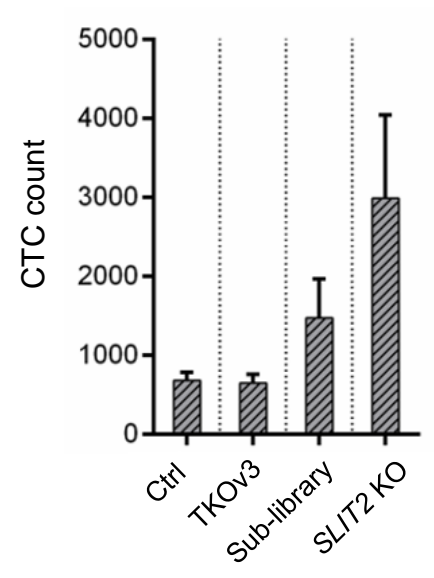

C

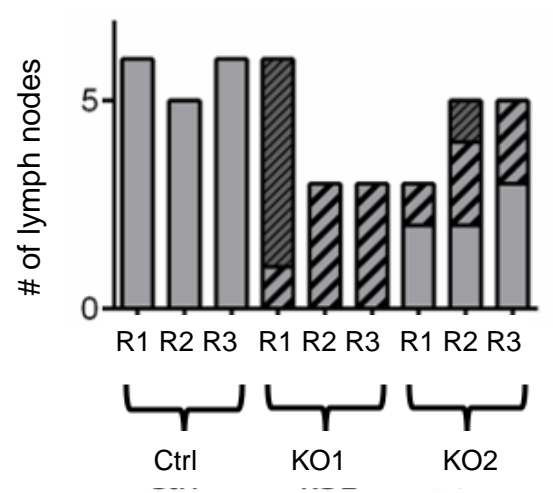

b
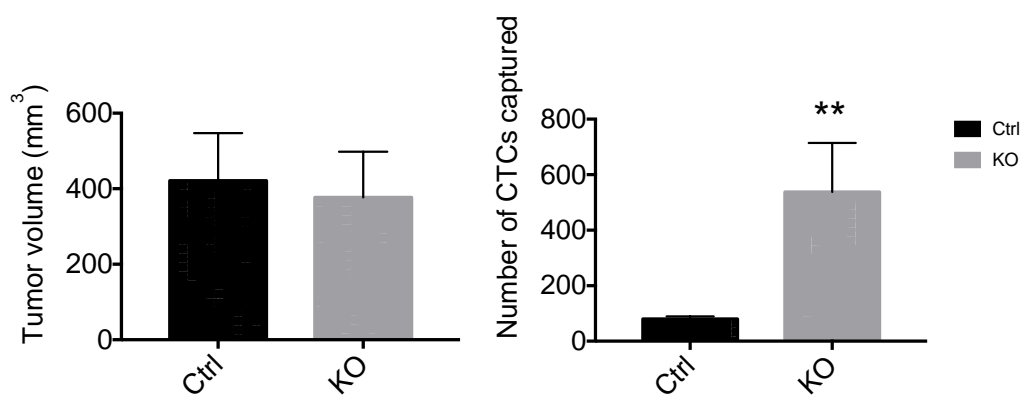

d

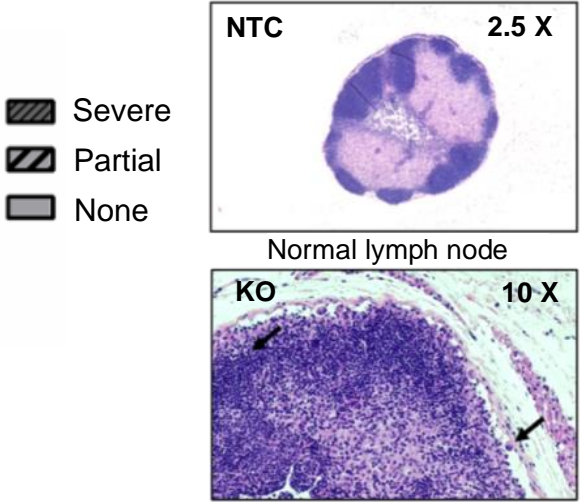

Subcapsular infiltration
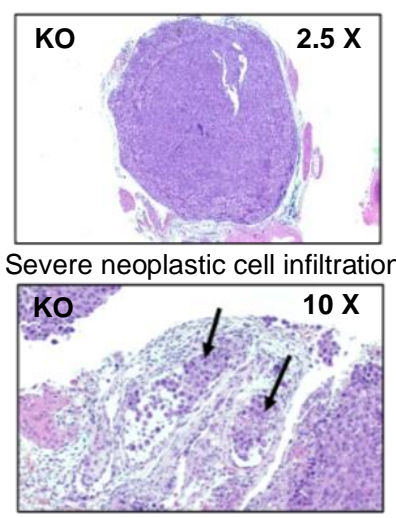

Intravascular infiltration

Figure 4: SLIT2 KO promoted cancer progression in vivo. a. Bar graph of the number of CTCs counted from the whole blood of the immunocompromised mice after transplant of non-targeted control, SLIT2 KO, or CRISPR library-transduced cells ( $n=3$ for each group). Error bars indicate SD. b. Bar graph comparing CTC number resulted from similar tumor size of NTC and SLIT2 KO cells $(n=3$ for each group). Error bars indicate SD. $P$ value was calculated by two-tailed unpaired t-test. $P=0.0072$. c. Bar graph of the number of lymph nodes with normal, neoplastic cell infiltrated, and severely infiltrated tissues. Histology slides were prepared from lymph nodes collected from the immunocompromised mice after orthotopic injection of either non-targeted control or the two SLIT2 KO cell lines. d. Representative images of the H\&E stained sections showing normal lymph node tissues (NTC), as well as severe, subcapsular, and intravascular neoplastic cell infiltration (SLIT2 KO). 
a

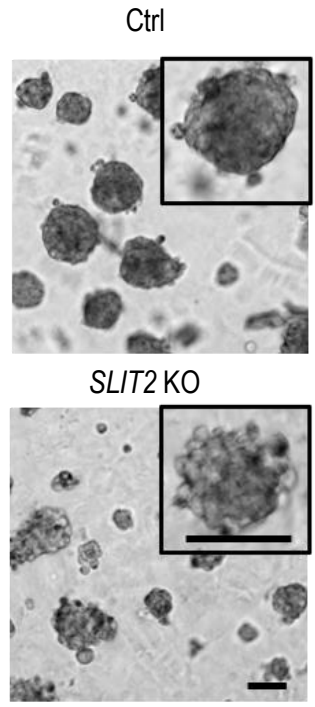

d

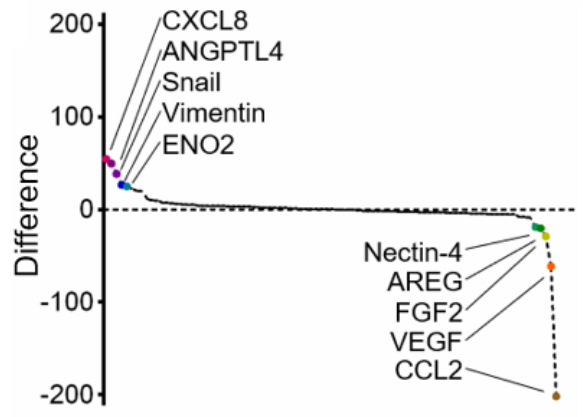

Antibody array (SLIT2 KO vs Ctrl) b

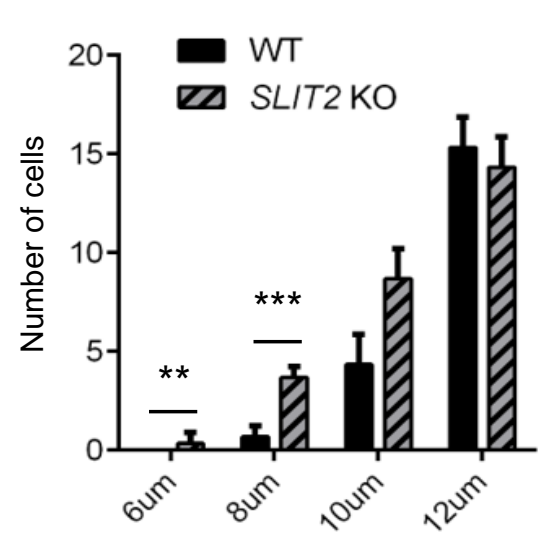

e

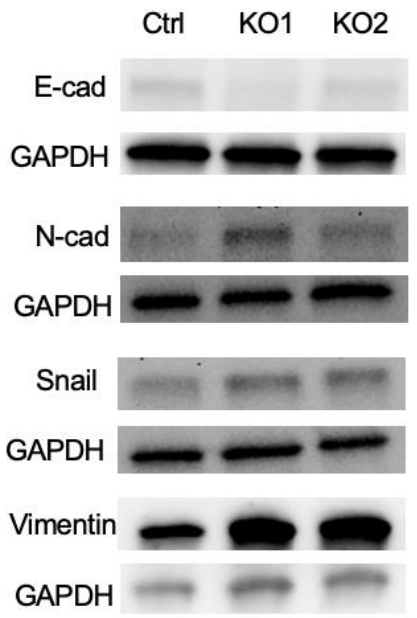

C

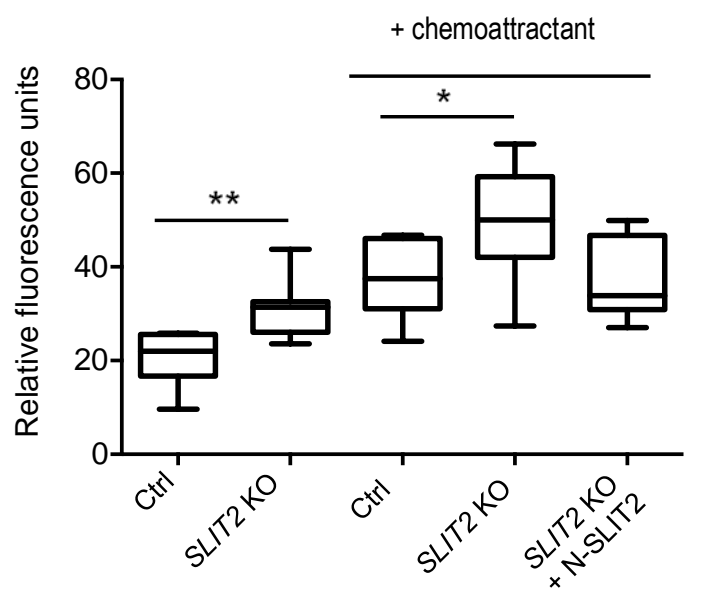

$\mathbf{f}$

PC-3M PC-3M

(Ctrl) (SLIT2 KO)

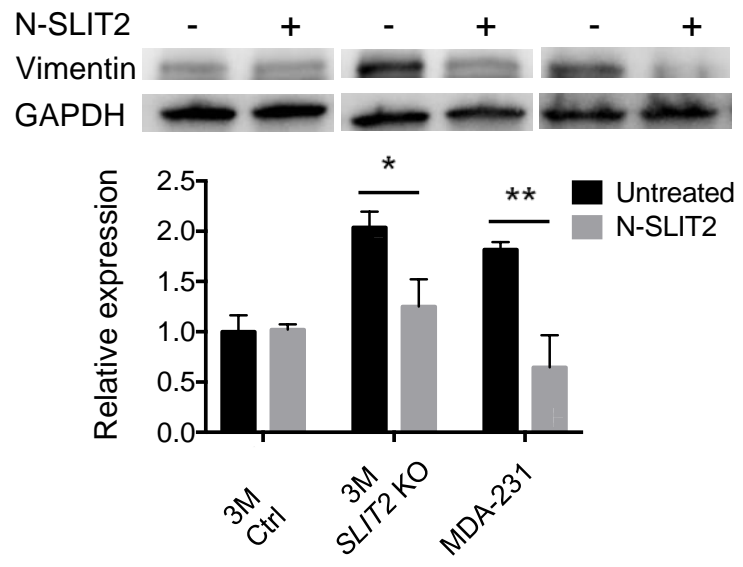

Figure 5: SLIT2 KO facilitated metastasis with activation of EMT. a. Representative images of the spheroids formed from non-target control (NTC) or SLIT2 KO PC-3M cells. b. Bar graph comparing the number of deformed NTC versus SLIT2 KO cells at different migration channel width $(\mathrm{n}=3$ for each group). c. Box plot comparing the number of NTC versus SLIT2 KO cells invaded across the transwell membrane with or without the presence of the chemoattractant or N-SLIT2 protein in the culture media ( $\mathrm{n}$ $=8$ for each group). d. Proteins ranked based on levels of differential expression in SLIT2 KO compared to NTC cells as detected by the oncology antibody array. e. Immunoblots detecting E-cadherin, Ncadherin, Snail, and Vimentin expression in SLIT2 KO and NTC cells. f. Immunoblots detecting Vimentin protein levels in control and SLIT2 KO PC-3M, as well as MDA-231 cells, either with or without supplementation of N-SLIT2 protein in culture medium. Error bars indicate SD. P values were calculated by two-way ANOVA, except for (c) which was by two-tailed unpaired t-test. ${ }^{* \star *} \mathrm{P}<0.001 ;{ }^{* \star} \mathrm{P}<0.01$; ${ }^{*} \mathrm{P}<0.05$; NS:P>0.05. 
a

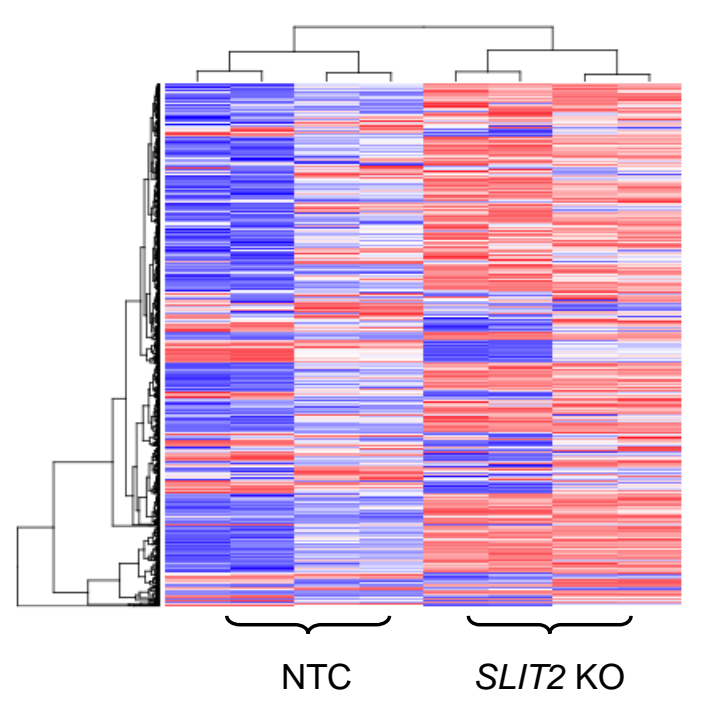

C

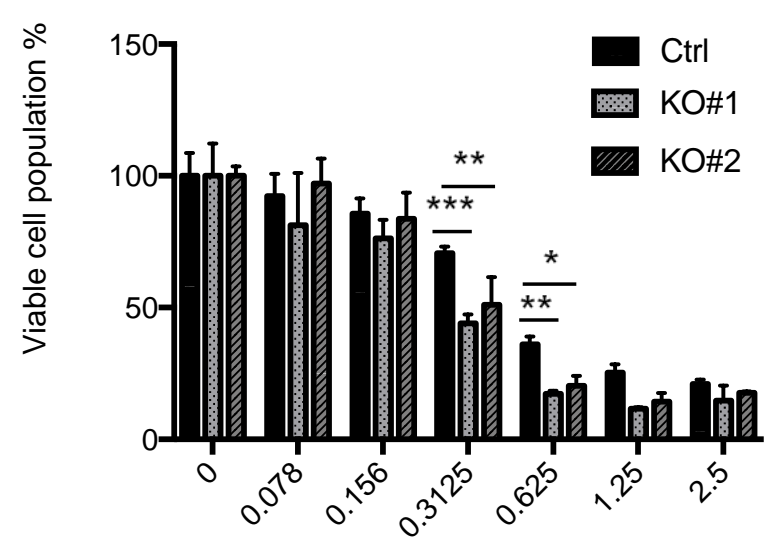

Rotenone $(\mu \mathrm{M})$ b

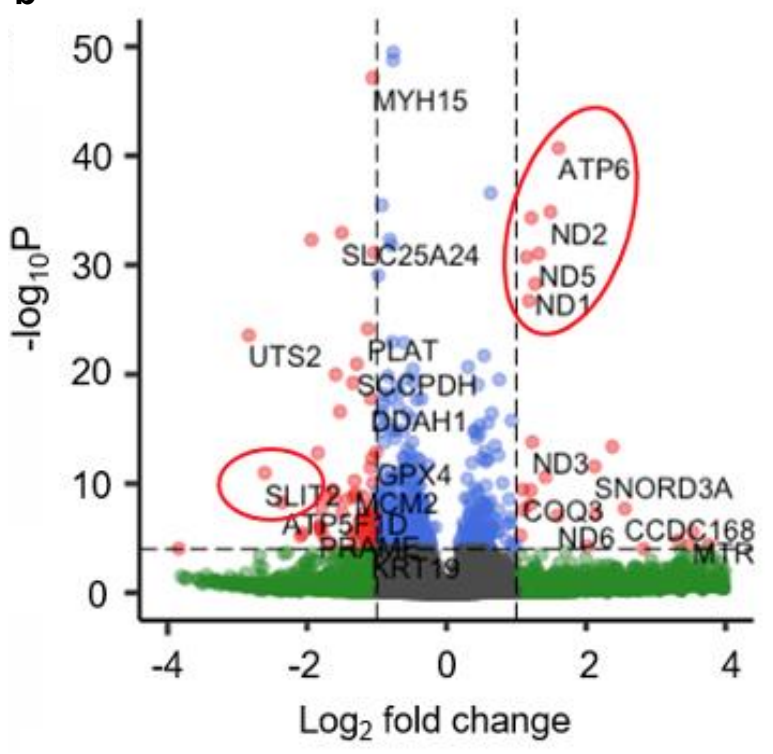

d

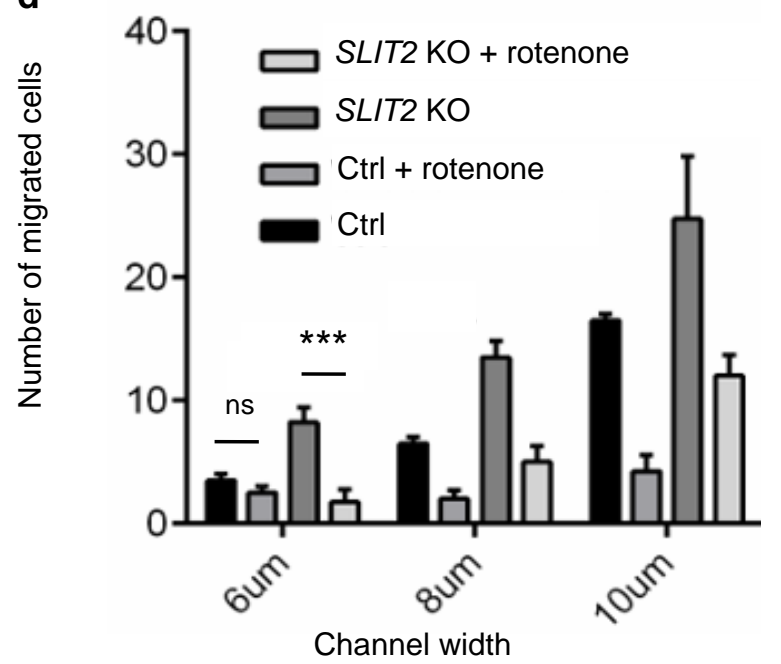

Figure 6: SLIT2 KO resulted in elevated complex I expression and hypersensitivity to rotenone. a. Heat map of the top 1000 differentially expressed genes in SLIT2 KO cells compared to the non-targeted control. b. Volcano plot highlighting (in red circles) the most differentially expressed genes of SLIT2 KO cells compared to NTC. c. Bar graph showing viability of the SLIT2 KO compared to NTC cells under titration of rotenone ( $n=3$ for each group). $d$. Bar graph showing the number of SLIT2 KO cells migrated across various channel width compared to the NTC cells, with or without the presence of rotenone at $\mathrm{IC}_{20}(\mathrm{n}=3$ for each group). Error bars indicate SD. $\mathrm{P}$ values were calculated by two-way ANOVA. ${ }^{* *} \mathrm{P}<0.001 ;{ }^{* *} \mathrm{P}<0.01 ;{ }^{*} \mathrm{P}<0.05 ; \mathrm{NS}: \mathrm{P}>0.05$. 